\title{
Interacciones medicamentosas de anti- infecciosos que desencadenan enfermedad renal: Aproximación para establecer y valorar su relevancia clínica. Revisión sistemática cualitativa
}

\author{
Mauricio Ceballos* \\ Johan Granados** \\ Pedro Amariles***
}

\begin{abstract}
*Químico Farmacéutico. Magister en Epidemiología. Profesor Asociado. Miembro del Grupo de investigación de Promoción y Prevención Farmacéutica. Grupo de investigación de Tecnología en Regencia de Farmacia. Facultad de Ciencias Farmacéuticas y Alimentarias. Universidad de Antioquia. Medellín. Colombia.

${ }^{* *}$ Químico Farmacéutico. Magister en Epidemiología. Miembro del Grupo de investigación de Promoción y Prevención Farmacéutica. Grupo de investigación de Tecnología en Regencia de Farmacia. Facultad de Ciencias Farmacéuticas y Alimentarias. Universidad de Antioquia. Medellín. Colombia.

***Químico Farmacéutico. Doctor en Farmacología. Profesor Titular Facultad de Ciencias Farmacéuticas y Alimentarias. Director del Grupo de investigación de Promoción y Prevención Farmacéutica. Facultad de Ciencias Farmacéuticas y Alimentarias. Universidad de Antioquia. Medellín. Colombia.

Correspondencia: Prof. Javier Mauricio Ceballos. Universidad de Antioquia. Facultad de Ciencias Farmacéuticas y Alimentarias. Calle 67 No. 53 - 108. Bloque 2, oficina 135. Medellín. Colombia. Correo electrónico: javier.ceballos@udea.edu.co
\end{abstract}

\section{RESUMEN}

Introducción: las interacciones medicamentosas de anti-infecciosos pueden desencadenar enfermedad renal; sin embargo, la información de este efecto es limitada, por tanto, la identificación, prevención y manejo de las interacciones medicamentosas clínicamente relevantes se considera un aspecto clave en la consecución de los objetivos terapéuticos en pacientes en tratamiento con anti-infecciosos. Objetivo: identificar y valorar la relevancia clínica de interacciones medicamentosas de anti-infecciosos que causan enfermedad renal. Metodología: revisión sistemática cualitativa de interacciones medicamentosas de anti-infecciosos asociadas a enfermedad renal. La relevancia clínica de las interacciones medicamentosas se valoró acorde con la probabilidad de ocurrencia y la gravedad del efecto. La búsqueda se realizó en la base de datos PubMed/Medline, de artículos publicados en inglés o español, entre agosto de 2006 y agosto de 2016, utilizando los siguientes términos Mesh y operadores boléanos: "Renal Insufficiency" OR "Anti-Infective Agents" OR "Antifungal Agents" OR "Anti-Bacterial Agents" AND "Drug Interactions" OR "Herb-Drug Interactions" OR "Food-Drug Interactions". Resultados: se identificaron 44 publicaciones y se incluyeron 9; en ellas, se identificaron 12 interacciones medicamentosas asociadas a enfermedad renal. Las combinaciones asociadas a enfermedad renal fueron: inhibidores de proteasa/nifedipino, cobicistat/fenofibrato/pravastatina, tenofovir/metformina, macrólidos/ estatinas, macrólidos/bloqueadores de los canales de calcio, quinolonas/warfarina, valaciclovir/loxoprofen y ácido fusídico/pravastatina. Conclusiones: los inhibidores de proteasa, macrólidos y quinolonas, al igual que el tenofovir, cobicistat, valaciclovir y ácido fusídico pueden generar enfermedad renal cuando se utilizan simultáneamente con otros medicamentos, en especial con estatinas, bloqueadores de canales de calcio, warfarina, metformina o loxoprofen. MÉD.UIS. 2017;30(3):101-9.

Palabras clave: Enfermedades renales. Anti-infecciosos. Antibacterianos. Interacciones de Drogas.

\section{Interactions of anti-infectious drugs that trigger renal disease: Approach to establish and assess its clinical relevance. Systematic qualitative review}

\section{ABSTRACT}

Introduction: drug interactions of anti-infectives can trigger renal disease; however, information on this effect is limited. Therefore, the identification, prevention and management of clinically relevant drug interactions are a key aspect in the achievement of therapeutic objectives in patients receiving anti-infectives. Objective: to identify and assess the clinical relevance of anti-infective drug interactions that causes kidney disease. Methodology: systematic qualitative review of drug interactions of anti-infectives associated with renal

Artículo recibido el 6 de mayo de 2017 y aceptado para publicación el 14 de julio de 2017 
disease. The clinical relevance of drug interactions was assessed according to the probability of occurrence and severity of the effect. The search was done in the PubMed/Medline database of articles published in english or spanish, between august 2006 and august 2016, using the following Mesh terms and Boolean Operators: "Renal Insufficiency" OR " Anti-Bacterial Agents "OR" Drug Interactions "OR" HerbDrug Interactions "OR” Food-Drug Interactions “. Results: we identified 44 publications and nine were included. In these nine articles, 12 drug interactions associated with renal disease were identified. Combinations associated with renal disease were protease inhibitors/ nifedipine, cobicistat/fenofibrate/pravastatin, tenofovir/metformin, macrolides/statins, statins/calcium channel blockers, quinolones/ warfarin, valaciclovir/loxoprofen and fusidic acid/Pravastatin. Conclusions: protease inhibitors, macrolides and quinolones, as well as tenofovir, cobicistat, valaciclovir and fusidic acid can generate renal disease when used simultaneously with other drugs, especially with statins, calcium channel blockers, warfarin, metformin or loxoprofen. MÉD.UIS. 2017;30(3):101-9

Keywords: Renal Insufficiency. Anti-Infective Agents. Anti-Bacterial Agents. Drug Interactions.

¿Cómo citar este artículo?: Ceballos M, Granados J, Amariles P. Interacciones medicamentosas de anti-infecciosos que desencadenan enfermedad renal: Aproximación para establecer y valorar su relevancia clínica. Revisión sistemática cualitativa. MÉD.UIS. 2017;30(3):101-9.

\section{INTRODUCCIÓN}

Las Interacciones Medicamentosas (IM) están asociadas a problemas de efectividad y seguridad del tratamiento, lo cual aumenta por la polimedicación y factores asociados al envejecimiento de la población. Existen reportes donde se evaluó la prevalencia de IM clínicamente relevantes en pacientes con infección por VIH, la mayoría de ellos en países desarrollados, en los cuales, la prevalencia osciló entre $27 \mathrm{y}$ $40 \%, 2$ situando las IM como una de las principales causas de los ingresos y aumento en las estancias hospitalarias. En este sentido, varios antibióticos han sido incluidos en la lista de medicamentos de alto riesgo publicada por el National Health Service3, considerados como los que presentan una mayor probabilidad de causar daños graves o incluso mortales a los pacientes, especialmente cuando se utilizan incorrectamente ${ }^{4}$. Además, con la aparición de microorganismos multirresistentes, las opciones terapéuticas se han visto limitadas y con ellas las posibilidades de logro de los objetivos terapéuticos, a lo que se suma que, el interés de la industria farmacéutica por la investigación y el desarrollo de nuevos antimicrobianos, es menor, asociado a una mayor prioridad por otros tipos de medicamentos 5 . Por tanto, es necesario optimizar la terapia con anti-infecciosos y garantizar un uso eficiente de los recursos disponibles.

El incremento de patógenos multirresistentes, sumado a la dificultad en la detección y el manejo de las IM, asociada a la complejidad de los esquemas terapéuticos, genera la necesidad de maximizar la efectividad de los anti-infecciosos, al igual que a minimizar los problemas de seguridad. Por tanto, la identificación, prevención y manejo de las IM clínicamente relevantes se considera un aspecto clave en la consecución de los objetivos terapéuticos en pacientes en tratamiento con anti-infecciosos. Entre los problemas de seguridad, asociadas a las IM, se encuentra la alteración o daño renal. Sin embargo, la información sistemática disponible de este tipo de interacción se considera limitada, aunque la alteración o daño renal inducida por medicamentos es un serio problema en la práctica clínica y representa entre el 8 y $60 \%$ de los casos con enfermedad renal aguda en pacientes hospitalizados ${ }^{6}$, siendo la reacción adversa más frecuente en pacientes que utilizan medicamentos anti-infecciosos, como se encontró en un reciente estudio, en el cual, alrededor del 18\% de los pacientes desarrollaron alteración o daño renal agudo durante su período de hospitalización al utilizar anti-infecciosos como aminoglucósidos, anfotericina B, vancomicina y antibióticos betalactámicos?. En este sentido, el objetivo de este trabajo fue identificar y valorar la relevancia clínica de las IM de los anti-infecciosos que desencadenan enfermedad renal.

\section{Materiales y MÉtodos}

Se realizó una revisión estructurada en PubMed/ Medline, de artículos publicados en inglés o español, con acceso a texto completo entre agosto de 2006 y agosto de 2016, sobre IM con anti-infecciosos que desencadenan enfermedad renal en humanos. La búsqueda se realizó con los siguientes términos Mesh y operadores booleanos: "Renal Insufficiency" $O R$ "Anti-Infective Agents" OR "Antifungal Agents" $O R$ "Anti-Bacterial Agents" AND "Drug Interactions" OR "Herb-Drug Interactions" OR "Food-Drug Interactions".

Los criterios de inclusión fueron: contener en el título o resumen información de interacciones de los anti-infecciosos con medicamentos, alimentos, 
fitoterapéuticos (plantas medicinales). Por su parte, los criterios de exclusión fueron: publicaciones con métodos in vitro o en animales, con fármacos en fase experimental y aquellos que no abordaron interacciones con medicamentos anti-infecciosos; artículos sin ninguna relación con los objetivos de la búsqueda y de la revisión; y artículos con información limitada para evaluar la relevancia clínica de la interacción (datos incompletos de los pacientes o de los medicamentos implicados en las IM, o del desenlace final de la IM).

Los artículos incluidos fueron seleccionados por tres investigadores de forma pareada. Para ello, se revisaron los títulos y resumenes de todos los artículos identificados para decidir su elegibilidad. Los artículos seleccionados se analizaron conjuntamente y, por consenso, se definió su inclusión o no.

\section{VALORACIÓN DE LA RELEVANCIA CLÍNICA}

La valoración de la relevancia clínica de las interacciones identificadas, se basó en la combinación de la gravedad del efecto de la interacción con la probabilidad de ocurrencia, de acuerdo al artículo publicado por Amariles $\mathrm{P}$, et al. Interacciones medicamentosas: aproximación para establecer y evaluar su relevancia clínica ${ }^{8}$, tal como se especifica a continuación:

\section{GRAVEDAD DE LA INTERACCIÓN}

1. Grave. La interacción puede causar daño o lesión al paciente. El resultado clínico negativo de la farmacoterapia puede generar la muerte del paciente, riesgo para la vida, hospitalización, una incapacidad permanente o significativa, anomalías congénitas o malformaciones al nacer, al igual que otros efectos que a juicio médico puedan comprometer la integridad del paciente y generar la necesidad de realizar una intervención quirúrgica para evitar la muerte, hospitalización o anomalías congénitas.

2. Moderada. La interacción genera la necesidad de realizar un seguimiento del paciente. El resultado clínico negativo de la farmacoterapia puede causar una modificación, cambio o interrupción de la farmacoterapia o el empleo de nuevos fármacos para tratar el problema relacionado con medicamentos, o bien la prolongación de la hospitalización del paciente.

3. Leve. La interacción no causa daño al paciente. El resultado negativo de la medicación no requiere la modificación (cambio o interrupción) de la farmacoterapia o el empleo de nuevos fármacos para tratar el problema relacionado con medicamentos ni prolonga la hospitalización del paciente.

\section{PROBABILIDAD DE APARICIÓN DE LA INTERACCIÓN}

Por su parte, la probabilidad de aparición de la interacción se establece en tres categorías: definida, probable o posible a partir del tipo de estudio que ha documentado la interacción y que ha sido publicado en revistas con revisión previa e indexada en bases de datos:

1. Definida: interacción documentada en metaanálisis, revisiones sistemáticas o ensayos clínicos aleatorizados o no aleatorizados.

2. Probable: interacción documentada en estudios analíticos o por la descripción de tres o más casos.

3. Posible: interacción documentada por la descripción de menos de tres casos o por recomendaciones de grupos de expertos.

A partir de las combinaciones posibles de gravedad y probabilidad de aparición, las interacciones pueden agruparse en cuatro categorías:

- Nivel 1: riesgo muy alto, resultante de la combinación grave y definida, o grave $y$ probable. En este caso el uso simultáneo de los medicamentos se considera absolutamente contraindicado.

- Nivel 2: riesgo alto, resultante de la combinación grave y posible, moderada y definida o moderada y probable. El uso simultáneo requiere ajustes en la dosificación y valoración clínica, idealmente de forma cuantitativa.

- Nivel 3: riesgo medio, resultante de la combinación moderada y posible, leve y definida o leve y probable. También es necesario un monitoreo clínico frecuente y un análisis sobre la necesidad de ajustar la dosis.

- $\quad$ Nivel 4: riesgo bajo, resultante de la combinación leve y posible. La interacción es de escasa relevancia clínica.

\section{CÁLCULO DE VALORES PROMEDIO DE CREATININA SÉRICA}

Utilizando los datos clínicos de creatinina sérica en los estudios analizados que lo reportaron, se calculó la diferencia promedio de creatinina 
sérica entre el inicio y el final de la exposición de los medicamentos o al momento de presentarse la enfermedad renal como consecuencia de la IM. Para ello, se halló el valor promedio de creatinina sérica al inicio y al final, y se calculó su diferencia. Adicionalmente, se estableció el promedio de días entre el inicio de la exposición y la aparición de la enfermedad renal.

\section{FORMATO RECOLECCIÓN DE INFORMACIÓN}

En Excel 2016 para Windows ${ }^{\circledR}$ se diseñó un formato para consignar y tabular los datos de las interacciones de los medicamentos, con la siguiente estructura: a) referencia bibliográfica; b) tipo de estudio; c) características de los sujetos (edad, peso, sexo, antecedentes clínicos); d) desenlaces clínicos secundarios; e) medicamento antiinfeccioso; f) grupo farmacológico del medicamento anti-infeccioso; g) otros medicamentos usados concomitantemente; h) grupo farmacológico de los otros medicamentos; i) nivel de creatinina al inicio; j) nivel de creatinina al final; k) tiempo de aparición del evento; I) clase de interacción (medicamentomedicamento, medicamento-fitoterapéutico, medicamento-alimento); m) mecanismo de la interacción (farmacocinética o farmacodinámica); n) nivel; gravedad y probabilidad de aparición de la interacción.

\section{Resultados}

En total, 44 referencias bibliográficas fueron identificadas y se seleccionaron nueve artículos, (Ver Figura 1). Se identificaron doce IM, de ellas, seis fueron valoradas como de riesgo muy alto (nivel 1) y seis de riesgo alto (nivel 2), todas asociadas a interacciones farmacocinéticas, principalmente relacionadas con el complejo enzimático Citocromo P-450 (CYP) (Ver Tabla 1).

\section{INTERACCIONES MEDICAMENTOSAS DE LOS ANTI- INFECCIOSOS CON DESENLACE DE ENFERMEDAD RENAL}

\section{Antirretrovirales inhibidores de proteasa, tenofovir y cobicistat}

En esta revisión se encontraron dos reportes de caso de interacciones asociadas a enfermedad renal entre estos medicamentos y estatinas, en especial atorvastatina, lovastatina y simvastatina, las cuales son sustrato de la CYP y sufren un metabolismo importante de primer paso, específicamente por la CYP3A4 ${ }^{9}$. El primero por la combinación atazanavir y simvastatina ${ }^{10}$ (relevancia nivel 2 ), y el otro por el uso simultaneo de cobicistat, fenofibrato y pravastatina" (relevancia nivel 2). Además, se encontró un reporte de caso de enfermedad renal asociado a la combinación de lopinavir/ritonavir y nifedipino ${ }^{12}$ (relevancia nivel 2), al igual que otro de enfermedad renal y acidosis láctica, atribuida a la utilización conjunta de tenofovir y metformina ${ }^{13}$ (relevancia nivel 2). El nivel de relevancia de las interacciones se detalla en la tabla 1.

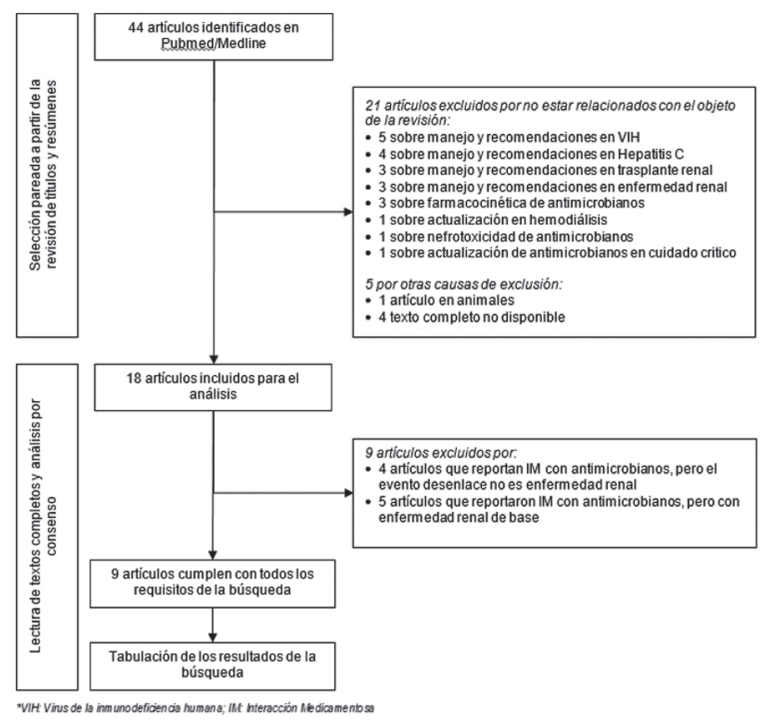

Figura 1. Proceso de identificación y Selección de artículos Fuente: Autores.

\section{Antivirales}

En el caso de la combinación de valaciclovir con analgésicos antiinflamatorios no esteroideos (AINEs), un análisis de reportes de forma retrospectiva del programa de farmacovigilancia de la Food and Drug Administration (FDA), identificó una asociación estadísticamente significativa del uso de loxoprofen y valaciclovir con el desarrollo de enfermedad renal en pacientes de edad avanzada ${ }^{14}$. Esta IM, acorde con su relevancia clínica se clasifica de nivel 1 (Ver Tabla 1 )

\section{Antibióticos macrólidos}

Un estudio de cohortes retrospectivo encontró asociación entre hospitalización por enfermedad renal aguda $o$ hipercalemia, al igual que con mortalidad por todas las causas, con la utilización conjunta de claritromicina, eritromicina o azitromicina con lovastatina, atorvastatina y simvastatina ${ }^{15}$ 
(relevancia nivel 1). Estos resultados fueron similares a los evidenciados en otro estudio de cohortes retrospectivo en personas de edad avanzada, que requirieron hospitalización por enfermedad renal (resultado primario) u hospitalización por hipotensión y muerte por cualquier causa (resultado secundario), eventos asociados a la combinación de un macrólido (claritromicina o azitromicina) con un blanqueador de canales de calcio (BBC) como amlodipino, felodipino, nifedipino, diltiazem $\mathrm{O}$ verapamilo $^{16}$ (relevancia nivel 1). El nivel de relevancia de estas 5 IM se detalla en la tabla 1.

\section{Antibióticos Quinolonas}

Un reporte de caso documentó la generación de enfermedad renal aguda, debido a coagulapatía grave, asociado a la combinación de levofloxacino y warfarina. ${ }^{17}$ El nivel de relevancia de esta IM es 2 (Ver Tabla 1).

\section{Otros antibióticos: Ácido fusídico}

Se identificó un reporte de caso de enfermedad renal aguda notoria con necrosis tubular aguda y rabdomiolisis atribuido a la combinación de ácido fusídico y pravastatina ${ }^{18}$. El nivel de relevancia de esta IM es 2 ( Ver Tabla 1).

\section{Discusión}

Los anti-infecciosos implicados en las IM asociados a enfermedad renal, en especial aguda, son de gran utilidad clínica en el ámbito hospitalario y ambulatorio. Los macrólidos y los antirretrovirales inhibidores de la proteasa IP fueron el grupo de anti-infecciosos con mayor frecuencia de IM que podrían generar enfermedad renal; ocho de las doce IM identificadas. La creatinina sérica fue el parámetro clínico de elección en todos los reportes de caso para medir el funcionamiento renal, excepto en uno de ellos, específicamente en el estudio que describe la IM entre valaciclovir y loxoprofen ${ }^{19}$, en el cual, el desenlace primario fue la falla renal aguda (no se describen valores de creatinina sérica u otro parámetro clínico) reportado como evento adverso, de acuerdo a la revisión retrospectiva de los reportes de la FDA. Acorde con los valores de creatinina sérica, el valor promedio inicial o basal de los once IM con el dato disponible fue de 1,2 mg/dL (DE 0,39 $\mathrm{mg} / \mathrm{dL}$ ); mientras que, al final de la exposición a los medicamentos o al momento de presentarse el evento renal fue de $6,6 \mathrm{mg} / \mathrm{dL}$ (DE 2,8 mg/dL). Por tanto, la diferencia promedio de creatinina entre el inicio y el final, fue de 5,4 mg/dL (DE 2,7 mg/dL). De igual forma, se encontró que la cantidad promedio de días entre el inicio de la exposición y la aparición de la enfermedad renal fue de 8,5 días (DE 4,1 días).

\section{Antirretrovirales inhibidores de proteasa, tenofovir y cobicistat}

Las isoenzimas del CYP3A son responsables del metabolismo de los inhibidores de proteasa, los cuales, a su vez, se comportan como inhibidores fuertes de la CYP3A. Por tanto, existe un importante potencial de IM de las farmacoterapias que incluyan un IP, mediada por la inhibición competitiva y reversible de la CYP3A4. Entre los IP más asociados a IM se encuentran el ritonavir y atazanavir. Adicionalmente, el cobicistat es un potente inhibidor de la $\mathrm{CYP}_{3} \mathrm{~A}_{4}$ que se utiliza como potenciador farmacocinético de otros antirretrovirales, como atazanavir o darunavir en el tratamiento de pacientes con VIH/SIDA ${ }^{20}$.

Un reporte de caso relacionado con la administración de nifedipino con ritonavir, mostró un aumento en las concentraciones de nifedipino, produciendo hipotensión e insuficiencia renal ${ }^{8}$, lo que se podría explicar por el efecto inhibidor de metabolismo de nifedipino por el ritonavir. Relacionado con este aspecto, es necesario mencionarque, en pacientescon VIH, cada vez es más común la utilización de fármacos hipolipemiantes, debido al aumento de la esperanza de vida de este grupo de pacientes, situación que se asocia a la presentación de otros problemas de salud, tales como aumento de riesgo cardiovascular, envejecimiento biológico acelerado, proceso inflamatorio crónico y la exposición prolongada a los medicamentos ${ }^{21}$; situación que puede asociarse a $I M$, debido a que varios antiretrovirales, en especial los IP, incluyendo ritonavir, lopinavir y atazanavir, al igual que el extensor farmacocinético cobicistat, pueden comportarse como inhibidores enzimáticos importantes del metabolismo de las estatinas. Por ello, en pacientes con VIH/SIDA se recomienda utilizar dosis bajas de estatinas, preferiblemente pravastatina, con fenofibrato el tratamiento, cuando se valore necesario.

Sin embargo, un caso identificado en esta revisión ${ }^{11}$ reportó rabdomiolisis y enfermedad renal por la combinación de pravastatina/fenofibrato y cobicistat. El mecanismo de esta IM se debe a que el cobicistat incrementa los niveles de pravastatina, lo que se 
podría atribuir a la capacidad de inhibir la proteína de resistencia de cáncer de mama (BCRP, por sus siglas en inglés), la glicoproteína-P y el transportador aniónico orgánico OATP1B1 hepático. Además, el cobicistat inhibe la secreción de creatinina por el túbulo proximal, que conduce a un ligero aumento de la creatinina sérica $(<10 \%)$ y una disminución de la filtración glomerular ${ }^{11}$. No obstante, es posible que otros factores hayan contribuido a favorecer la enfermedad renal, como la edad, la nefrotoxicidad por fenofibrato, la hipertensión arterial y la misma infección por el VIH.

En esta revisión se identificó un reporte de caso de enfermedad renal asociada a acidosis láctica por la utilización conjunta de metformina y tenofovir ${ }^{13}$. Desde una perspectiva farmacológica, esta IM podría deberse a una sinergia de efectos adversos de estos dos fármacos (mecanismo farmacodinámico). En este sentido, el tenofovir se ha asociado a acidosis láctica, esteatosis hepática (hígado graso), neuropatía periférica y pancreatitis ${ }^{13}$. Por su parte, la metformina debe su efecto terapéutico a su capacidad de inhibir la gluconeogénesis hepática y de aumentar la sensibilidad muscular y del tejido adiposo a la insulina ${ }^{22}$; mientras que la acidosis láctica, especialmente no asociada a hipoxia hística o tipo B (la A se asocia con hipoxia hística) es una de sus reacciones adversas. Aunque la acidosis láctica por metformina presenta una alta mortalidad (30-50\% de los casos), su incidencia es muy rara, alcanzando sólo los 0,015 casos fatales/1000 pacientes al año) $)^{23,24}$.

\section{Antivirales}

El valaciclovir, aunque generalmente es bien tolerado, se ha asociado esporádicamente con el desarrollo de falla renal, principalmente en personas de edad avanzada, independiente de la presencia o no de enfermedad renal de base ${ }^{19,25}$. Se identificó una IM entre el valaciclovir (profármaco del Aciclovir) y el loxoprofen, un AINEs ${ }^{14}$, la cual podría atribuirse a que el valaciclovir es un substrato de los transportadores aniónicos orgánicos 1, 2 y 3 (OAT1, OAT2, OAT3, por sus siglas en inglés), responsables de su secreción tubular. Por tanto, la inhibición de los OAT1 y OAT3 por parte del loxoprofen ${ }^{26}$, podría generar un incremento en las concentraciones y efectos renales del valaciclovir. Adicionalmente, los AINEs, debido a sus efectos farmacológicos pueden favorecer la aparición de toxicidad renal. En esencia, este grupo de medicamentos disminuye la producción de prostaglandinas E2 e I2, lo que limita la vasodilatación y, con ello, reduce el flujo sanguíneo y la tasa de filtración glomerular, causando retención de sodio ${ }^{27}$, lo que genera un efecto sinérgico en la aparición de enfermedad renal aguda. Por tanto, esta interacción podría ser de mecanismos farmacocinético y farmacodinámico. El valaciclovir se ha asociado, esporádicamente a falla renal aguda, evento que es independiente de la presencia o no de enfermedad renal de base. Relacionado con este aspecto, es necesario destacar la importancia de ajustar la dosis de este antiviral, en pacientes con enfermedad renal crónica. El no seguimiento de esta recomendación aumenta la probabilidad de problemas de seguridad, incluyendo la falla renal y especialmente alteraciones neurológicas principalmente convulsiones ${ }^{28}$.

\section{Antibióticos macrólidos}

Los antibióticos macrólidos claritromicina y eritromicina son inhibidores fuertes de la enzima CYP3A4, mientras que, este efecto, es mínimo en el caso de la azitromicina. En los adultos mayores, la coadministración de claritromicina o eritromicina y una estatina metabolizada por la CYP3A4 (atorvastatina, simvastatina y lovastatina) se ha asociado con un mayor riesgo de hospitalización por enfermedad renal, e incluso de mortalidad, en comparación que con la coadministración de azitromicina. En este sentido, uno de los dos estudios analíticos, identificados en esta revisión, encontró que, en adultos mayores, la utilización concomitante de Bloqueadores de los Canales de Calcio (BCC) y claritromicina aumentó el riesgo de enfermedad renal. Relacionado con esta IM, es importante destacar que. 1) el riesgo fue menor con el uso de azitromicina y 2) los resultados soportan las recomendaciones de varios autores de evitar el uso simultáneo de BCC con inhibidores fuertes de la $\mathrm{CYP}_{3} \mathrm{~A}_{4}$, caso de los macrólidos ${ }^{15}$. En este sentido, los BCC son metabolizados por la CYP3A4 y, por tanto, la coadministración de los inhibidores de esta isoenzima pueden aumentar las concentraciones de los BCC de forma significativa (hasta en un $500 \%$ ) y, con ello, sus problemas de seguridad ${ }^{29}$. 
Tabla 1. Interacciones medicamentosas de agentes antiinfecciosos que desarrollan enfermedad renal

\begin{tabular}{|c|c|c|c|c|c|c|c|c|c|c|}
\hline $\begin{array}{c}\text { Grupo } \\
\text { farmacológico } \\
\text { del anti- } \\
\text { infeccioso }\end{array}$ & $\begin{array}{l}\text { Medicamento } \\
\text { concomitante }\end{array}$ & $\begin{array}{c}\text { Grupo } \\
\text { farmacológico } \\
\text { medicamento } \\
\text { concomitante }\end{array}$ & $\begin{array}{c}\text { Creatinina } \\
\text { (inicio) }\end{array}$ & $\begin{array}{c}\text { Creatinina } \\
\text { (final) }\end{array}$ & $\begin{array}{c}\text { Tiempo } \\
\text { aparición } \\
\text { del evento }\end{array}$ & $\begin{array}{c}\text { Tipo de } \\
\text { Interacción }\end{array}$ & $\begin{array}{c}\text { Mecanismo } \\
\text { de la } \\
\text { Interacción }\end{array}$ & Nivel & Gravedad & Probabilidad \\
\hline $\begin{array}{c}\text { Antirretroviral } \\
\text { Inhibidor de } \\
\text { proteasa }\end{array}$ & Simvastatina & Estatina & $0,9 \mathrm{mg} / \mathrm{dL}$ & $4,1 \mathrm{mg} / \mathrm{dL}$ & 9 días & $\begin{array}{l}\text { Medicamento - } \\
\text { Medicamento }\end{array}$ & FC & 2 & Grave & Posible \\
\hline $\begin{array}{c}\text { Antirretroviral } \\
\text { extensor } \\
\text { farmacocinético }\end{array}$ & $\begin{array}{l}\text { Pravastatina/ } \\
\text { fenofibrato }\end{array}$ & Estatina/fibrato & $1,16 \mathrm{mg} / \mathrm{dL}$ & $11,9 \mathrm{mg} / \mathrm{dL}$ & 14 días & $\begin{array}{l}\text { Medicamento - } \\
\text { Medicamento }\end{array}$ & FC & 2 & Grave & Posible \\
\hline Antirretroviral & Nifedipino & $\begin{array}{l}\text { Bloqueador de los } \\
\text { canales de calcio }\end{array}$ & $1,7 \mathrm{mg} / \mathrm{dL}$ & $7 \mathrm{mg} / \mathrm{dL}$ & 2 días & $\begin{array}{l}\text { Medicamento - } \\
\text { Medicamento }\end{array}$ & $\mathrm{FC}$ & 2 & Grave & Posible \\
\hline $\begin{array}{c}\text { Antirretroviral } \\
\text { inhibidor de } \\
\text { transcritasa } \\
\text { inversa análogo } \\
\text { de nucleótido }\end{array}$ & Metformina & $\begin{array}{l}\text { Antidiabético } \\
\text { (Biguanida) }\end{array}$ & $1,1 \mathrm{mg} / \mathrm{dL}$ & $6,3 \mathrm{mg} / \mathrm{dL}$ & 10 días & $\begin{array}{l}\text { Medicamento - } \\
\text { Medicamento }\end{array}$ & FD & 2 & Grave & Posible \\
\hline Antiviral & Loxoprofen & AINE & NR & NR & NR & $\begin{array}{l}\text { Medicamento - } \\
\text { Medicamento }\end{array}$ & $\mathrm{FC} / \mathrm{FD}$ & 1 & Grave & Probable \\
\hline Macrólido & $\begin{array}{l}\text { lovastatina, } \\
\text { storvastatina, } \\
\text { simvastatina }\end{array}$ & Estatina & NR & $\begin{array}{l}\text { Criterio } \\
\text { clínico* }\end{array}$ & NR & $\begin{array}{l}\text { Medicamento - } \\
\text { Medicamento }\end{array}$ & FC & 1 & Grave & Probable \\
\hline Mácrolido & $\begin{array}{l}\text { lovastatina, } \\
\text { storvastatina, } \\
\text { simvastatina }\end{array}$ & Estatina & NR & $\begin{array}{l}\text { Criterio } \\
\text { clínico* }\end{array}$ & NR & $\begin{array}{l}\text { Medicamento - } \\
\text { Medicamento }\end{array}$ & FC & 1 & Grave & Probable \\
\hline Mácrolido & $\begin{array}{l}\text { lovastatina, } \\
\text { storvastatina, } \\
\text { simvastatina }\end{array}$ & Estatina & NR & $\begin{array}{l}\text { Criterio } \\
\text { clínico* }\end{array}$ & NR & $\begin{array}{l}\text { Medicamento - } \\
\text { Medicamento }\end{array}$ & $\mathrm{FC}$ & 1 & Grave & Probable \\
\hline Mácrolido & $\begin{array}{l}\text { Amlodipino, } \\
\text { Dialtiazem, } \\
\text { Felodipino, } \\
\text { Nifedipino, } \\
\text { Verapamilo }\end{array}$ & $\begin{array}{l}\text { Bloqueador de los } \\
\text { canales de calcio }\end{array}$ & NR & $\begin{array}{l}\text { Criterio } \\
\text { clínico* }\end{array}$ & NR & $\begin{array}{l}\text { Medicamento - } \\
\text { Medicamento }\end{array}$ & $\mathrm{FC}$ & 1 & Grave & Probable \\
\hline Mácrolido & $\begin{array}{l}\text { Amlodipino, } \\
\text { Dialtiazem, } \\
\text { Felodipino, } \\
\text { Nifedipino, } \\
\text { Verapamilo }\end{array}$ & $\begin{array}{l}\text { Bloqueador de los } \\
\text { canales de calcio }\end{array}$ & NR & $\begin{array}{l}\text { Criterio } \\
\text { clínico* }\end{array}$ & NR & $\begin{array}{l}\text { Medicamento - } \\
\text { Medicamento }\end{array}$ & $\mathrm{FC}$ & 1 & Grave & Probable \\
\hline Quinolonas & Warfarina & $\begin{array}{c}\text { Anticoagulante } \\
\text { oral }\end{array}$ & $1,6 \mathrm{mg} / \mathrm{dL}$ & $6,2 \mathrm{mg} / \mathrm{dL}$ & 6 días & $\begin{array}{l}\text { Medicamento - } \\
\text { Medicamento }\end{array}$ & $\mathrm{FC} / \mathrm{FD}$ & 2 & Grave & Posible \\
\hline $\begin{array}{c}\text { Antibiótico } \\
\text { inhibidor de la } \\
\text { síntesis proteica } \\
\text { bacteriana }\end{array}$ & Pravastatina & Estatinas & $0,7 \mathrm{mg} / \mathrm{dL}$ & $4,5 \mathrm{mg} / \mathrm{dL}$ & 6 días & $\begin{array}{l}\text { Medicamento - } \\
\text { Medicamento }\end{array}$ & FC & 2 & Grave & Posible \\
\hline
\end{tabular}

* $0,3 \mathrm{mg} / \mathrm{dL}$ o más desde la línea de base o un incremento relativo del $50 \%$ o más

NR: No reportado; H: Hombre; M: Mujer; HTA: Hipertensión Arterial; VIH: Virus de la Inmunodeficiencia Humana; EPOC: Enfermedad Pulmonar Obstructiva Crónica; AINE: Analgésico antiiflamatorio no esteroideo; FC: Farmacocinético; FD: Farmacodinámico.

Fuente: Autores. 


\section{Antibióticos Quinolonas}

El levofloxacino podría inhibir el crecimiento de las bacterias entéricas productoras de vitamina $\mathrm{K}$, lo que podría aumentar el efecto anticoagulante de la warfarina. En este sentido, el levofloxacino se asoció el aumento del tiempo de protrombina y de la Razón o Relación Internacional Normalizada (INR, por sus siglas en inglés). En este caso, el autor atribuyó este aumento a reducción de la flora intestinal productora de vitamina K y la coagulapatía se asoció con enfermedad renal aguda ${ }^{17}$. Este efecto también se evidenció con el ofloxacino; pero en este caso el efecto se atribuyó a que el ofloxacino compite por su unión a la albumina, causando un aumento en la concentración de warfarina libre ${ }^{30}$. Este mecanismo, sumado a la posible disminución de la flora productora de vitamina $\mathrm{K}^{31}$, podría explicar la IM observada entre la warfarina y el levofloxacino. Aunque estos dos últimos reportes no fueron incluidos en los análisis de esta revisión por estar por fuera del periodo de tiempo utilizado como criterio de búsqueda, refuerzan la importancia de esta IM, al tiempo que permiten establecer que, el mecanismo de la IM entre las quinolonas con la warfarina, podría ser tanto farmacocinético como farmacodinámico.

\section{Otros antibióticos: Ácido fusídico}

El ácido fusídico puede causar un aumento notorio de la bilirrubina, asociado a posible esteatosis. Además, su administración conjunta con estatinas genera un aumento significativo de las concentraciones plasmáticas de ambos agentes ${ }^{32}$. De tal manera que ocurre un aumento significativo en el efecto de ambos agentes; incluyendo toxicidad renal e incluso rabdomiolisis. Acorde con la información disponible, el mecanismo de esta IM no está claramente establecido ${ }^{18}$.

Relacionado con la relevancia clínica de las interacciones, debido a que el desenlace es grave, resulta ser de nivel 102 . En el primer caso (nivel 1) por la combinación de grave con probable, caso de los estudios analíticos (casos y controles o cohorte) y en el segundo (nivel 2) por la combinación grave con posible, caso de los reportes de casos (inferiores a 3), tal como se detalla en la tabla 1. Este resultado es clave, debido a que muestra la necesidad de considerar este tipo de interacciones en la práctica clínica.
Las limitaciones principales de este estudio se deben a que la búsqueda se limitó a la base de datos PubMed/Medline. Sin embargo, este efecto pudo disminuirse debido a que la revisión se complementó con la inclusión de las referencias bibliográficas de los artículos consideradas de interés para el tema. Adicionalmente, la revisión de solo título y resumen en la primera etapa, al igual que la no revisión de la literatura gris pudo limitar identificar más trabajos de interés para el objeto de la revisión. En otros trabajos similares sería deseable la inclusión de literatura gris, utilizando una fuente como Google Académico.

\section{Conclusiones}

La enfermedad renal, en especial aguda, podría aparecer como consecuencia de la utilización simultánea de anti-infecciosos de tipo antirretrovirales inhibidores de proteasa, tenofovir, cobicistat y antivirales como el valaciclovir, al igual que de antibióticos macrólidos, quinolonas y ácido fusídico, con otros medicamentos, en especial de los grupos terapéuticos estatinas y bloqueadores de los canales de calcio o con warfarina, metformina o loxoprofen como fármacos específicos. De las 12 IM identificadas, seis son de nivel 1 y seis de nivel 2 , en esencia por el desenlace establecido de enfermedad renal aguda, el cual es un efecto grave en la salud de los pacientes.

\section{ReferenCIAS BiblográficAS}

1. Manzardo C, Tuset M, Miró JM, Gatell JM. Severe or lifethreatening interactions between antiretrovirals and non-HIV drugs. Enferm Infecc Microbiol Clin. 2015;33(7):15-30.

2. Martínez-Múgica, C. Potential antimicrobial drug interactions in clinical practice: consequences of polypharmacy and multidrug resistance. Rev Esp Quimioter. 2015;28(6):282-8.

3. Model of Care Polypharmacy Working Group. Polypharmacy Guidance. Scotland: Quality and Efficiency Support Team \& Scottish Government Health and Social Care Directorates; 2012. $47 \mathrm{p}$.

4. Institute for Safe Medication Practices. ISMP's list of high-alert medications. Pennsylvania. 2007.

5. Organización Mundial De La Salud. Estrategia mundial de la OMS para contener la resistencia a los antimicrobianos. 2001. $104 \mathrm{p}$.

6. Mizokami F, Mizuno T. Acute Kidney Injury Induced by Antimicrobial Agents in the Elderly: Awareness and Mitigation Strategies. Drugs Aging. 2015;32(1):1-12.

7. Khalili H, Bairami S, Kargar M. Antibiotics induced acute kidney injury: incidence, risk factors, onset time and outcome. Acta Med Iran. 2013;51(12):871-8.

8. Amariles P, Giraldo NA, Faus MJ. Interacciones medicamentosas: aproximación para establecer y evaluar su relevancia clínica. Med Clin (Barc). 2007;129(1):27-35.

9. Franco D, Henao Y, Monsalve M, Gutiérrez F, Hincapie J, Amariles P. Hypolipidemic agents drug interactions: approach to establish 
and assess its clinical significance. Structured review. Farm Hosp. 2013;37(6):539-57

10- Schmidt GA, Hoehns JD, Purcell JL, Friedman RL, Elhawi Y. Severe rhabdomyolysis and acute renal failure secondary to concomitant use of simvastatin, amiodarone, and atazanavir. J Am Board Fam Med. 2007;20(4):411-6.

11. Suttels V, Florence E, Leys J, Vekemans M, Van den Ende J, Vlieghe E, et al. A 68-year old male presenting with rhabdomyolysisassociated acute kidney injury following concomitant use of elvitegravir/cobicistat/emtricitabine/tenofovir disoproxil fumarate and pravastatin/fenofibrate: a case report. J Med Case Rep. 2015;9:190.

12. Baeza MT, Merino E, Boix V, Climent E. Nifedipine-lopinavir/ ritonavir severe interaction: a case report. AIDS. 2007;21(1):119-20.

13. Aperis G, Paliouras C, Zervos A, Arvanitis A, Alivanis P. Lactic acidosis after concomitant treatment with metformin and tenofovir in a patient with HIV infection. J Ren Care. 2011;37(1):25-9.

14. Yue Z, Shi J, Jiang P, Sun H. Acute kidney injury during concomitant use of valacyclovir and loxoprofen: detecting drug-drug interactions in a spontaneous reporting system. Pharmacoepidemiol. Drug Saf. 2014;23(11):1154-9.

15. Patel AM, Shariff S, Bailey DG, Juurlink DN, Gandhi S, Mamdani M, et al. Statin toxicity from macrolide antibiotic coprescription: a population-based cohort study. Ann Intern Med. 2013;158(12):869-76.

16. Gandhi S, Fleet JL, Bailey DG, McArthur E, Wald R, Rehman F, et al. Calcium-channel blocker-clarithromycin drug interactions and acute kidney injury. JAMA. 2013;310(23):2544-53.

17. Nemoto C, Ikegami Y, Shimada J, Tsukada Y, Abe Y, Tase C. Acute renal failure caused by severe coagulopathy induced by the interaction between warfarin potassium and levofloxacin: a case report. J. Anesth. 2012;26(6):943-4.

18. Bachoumas K, Fiancette M, Lascarrou JB, Lacherade JC, Leclair F Reignier J. Fatal rhabdomyolysis following the co-prescription of fusidic acid and pravastatin. Med Mal Infect. 2015;45(10):417-9.

19 Sugimoto T, Yasuda M, Sakaguchi M, Koyama T, Uzu T, Kashiwagi A, et al. Oliguric acute renal failure following oral valacyclovir therapy. QJM. 2008;101(2):164-6.
20. Temesgen Z. Cobicistat, a pharmacoenhancer for HIV treatments. Drugs Today (Barc). 2013;49(4):233-7.

21. Negredo E, Rey-Joly C. Cardiovascular risk and human immunodeficiency virus infection: A new clinical challenge. Med Clin (Barc). 2010;135(5):209-11.

22. Fisman EZ, Tenenbaum A. A cardiologic approach to non-insulin antidiabetic pharmacotherapy in patients with heart disease. Cardiovasc Diabetol. 2009;8:38.

23. Chang CT, Chen YC, Fang JT, Huang CC. Metformin-associated lactic acidosis: case reports and literature review. J Nephrol. 2002;15(4):398-402.

24. Rocha A, Almeida M, Santos J, Carvalho A. Metformin in patients with chronic kidney disease: strengths and weaknesses. J Nephrol. 2013;26(1):55-60.

25. Sagawa N, Tsurutani Y, Nomura K, Okuyama T, Kondo M, Sata A, et al. Acyclovir-induced neurotoxicity and acute kidney injury in an elderly diabetic patient treated with valacyclovir: report of a case. Nihon Ronen Igakkai Zasshi. 2014;51(6):581-5.

26. Uwai Y, Taniguchi R, Motohashi H, Saito H, Okuda M, Inui K. Methotrexate-loxoprofen interaction: involvement of human organic anion transporters hOAT1 and hOAT3. Drug Metab Pharmacokinet. 2004;19(5):369-74.

27. Morgan T, Anderson A. The effect of nonsteroidal antiinflammatory drugs on blood pressure in patients treated with different antihypertensive drugs. J Clin Hypertens (Greenwich). 2003;5(1):53-7.

28. Rodríguez JJ, Franco E, Delgado F, Villalobos F. Epilepticus status and valaciclovir in chronic renal failure. Med Clin (Barc). 2004;123(10):399.

29. Glesby MJ, Aberg JA, Kendall MA, Fichtenbaum CJ, Hafner R, Hall S, et al. Pharmacokinetic interactions between indinavir plus ritonavir and calcium channel blockers. Clin Pharmacol Ther. 2005;78(2):143-53

30. Leor J, Matetzki S. Ofloxacin and warfarin. Ann Intern Med. 1988;109(9):761.

31. Jones CB, Fugate SE. Levofloxacin and warfarin interaction. Ann Pharmacother. 2002;36(10):1554-7.

32. García-Rodríguez, J. A., Zufiaurre, N. G., \& Bellido JM. Ácido fusídico. Rev Esp Quimioter. 2003;16(2):161-71. 